\title{
Impact of Ice Slurry Ingestion During Break-Times on Repeated-Sprint Exercise in the Heat
}

\section{(ㄷ) (1) $(\mathrm{B})$}

\author{
Authors \\ Takashi Naito', Miki Haramura², 3, Koji Muraishi³, ${ }^{3}$, Misa Yamazaki ${ }^{3}$, Hideyuki Takahashi ${ }^{1}$
}

\section{Affiliations}

1 Department of Sports Research, Japan Institute of Sports Sciences, Kita-ku, Japan

2 Faculty of Sport Sciences, Waseda University, Shinjuku-ku,Japan

3 Department of Sports Sciences, Japan Institute of Sports Sciences, Kita-ku, Japan

4 Graduate School of Community and Health Services, Rikkyo University, Niiza-city, Japan

Key word

cooling, rectal temperature, work done, forehead skin temperature, perceptual sensations
received $\quad 11.11 .2019$
revised 20.02.2020
accepted $\quad 08.03 .2020$

Bibliography

DOI https://doi.org/10.1055/a-1139-1761

Sports Medicine International Open 2020; 4: E45-E52

(c) Georg Thieme Verlag KG Stuttgart · New York

ISSN 2367-1890

\author{
Correspondence \\ Takashi Naito \\ 3-15-1 Nishigaoka \\ Kita-ku \\ 115-0056 \\ Japan \\ Tel.: + 81359630231 , Fax : + 81359630232 \\ taka.1226n@gmail.com
}

Department of Sports Research, Japan Institute of Sports Sciences

\begin{abstract}
The study aimed to investigate the effects of ice slurry ingestion during break times and half-time (HT) on repeated-sprint performance and core temperature in the heat. Seven males performed two different trials as follows: ice slurry $\left(-1^{\circ} \mathrm{C}\right)$ or room temperature water ingestion at each break and $\mathrm{HT}$ break at $36.5^{\circ} \mathrm{C}, 50 \%$ relative humidity. Participants performed 30 sets of 1-min periods of repeated-sprint exercises protocol using a cycling ergometer. Each period consisted of $5 \mathrm{sec}$ of maximal pedaling, $25 \mathrm{sec}$ of pedaling with no workload, and $30 \mathrm{sec}$ of rest; two sets of exercise periods were separated by 10 min of rest. Each break was implemented for 1 min after every 5 sets. The rectal temperature in ice slurry ingestion was significantly lower than that of the room temperature water at 45 set $(p=0.04)$. Total and mean work done was greater in ice slurry ingestion compared to room temperature water ingestion $(\mathrm{p}<$ 0.05 ). These results suggested that ice slurry ingestion during break times and HT break may be an effective cooling strategy to attenuate the rise of core temperature in the second half of exercise and improve the repeated-sprint exercise capacity in the heat.
\end{abstract}

\section{Introduction}

Major international sporting competitions, such as the 2020 Olympics in Tokyo, are often held in hot conditions. Research on cooling the body to prevent a decrease in exercise performance in the heat has increased. The ingestion of an ice slurry or crushed ice has recently been proposed to minimize exercise performance impairments [1] and prevent hyperthermia [2,3]. Ice slurry ingestion before exercise is based on evidence that reducing one's initial core temperature allows for a greater heat storage capacity during exercise and in turn prolongs the onset of hyperthermia-induced fatigue [4-6]. Previous studies have reported that ingestion of ice slurry before exercise caused an approximately $0.5^{\circ} \mathrm{C}$ reduction in rectal temperature (Tre) and 16-19\% improvements in endurance exercise capacity compared with cold water ingestion in hot environments $\left(34.0-35.0^{\circ} \mathrm{C}, 50.0-55.9 \%\right.$ relative humidity) $[3,7]$. 
Researchers have previously experimented with ice slurry ingestion during exercise to investigate the effects of endurance exercise performance [8]. The aim of body cooling during exercise (called mid-cooling) is to reduce thermoregulatory strain during exercise by means of attenuating the rise of core temperature [9]. However, the ingestion of ice slurry during exercise may be more difficult during intense exercise with high ventilation compared with cold water, which is more easily consumed even though it is less effective in lowering core temperature [10]. Sports such as soccer or tennis, which include some break times and a half-time (HT) break, provide the opportunity to ingest a large amount of ice slurry, which is unlikely during the exercise. Stanley et al. [11] showed that the ingestion of ice slurry during $\mathrm{HT}$ reduced Tre until only 10 min after the start of the time-trial and did not improve in following time-trials compared to that of cold liquid ingestion. Participants in these studies ingested approximately $1000 \mathrm{~g}$ ice slurry. Ingesting ice slurry at HT only may not improve performance by a heat-sink effect on exercise performance. In contrast, Naito et al. [12] demonstrated that ice slurry ingestion $\left(1.25 \mathrm{~g} \cdot \mathrm{kg}\right.$ body mass $\left.[\mathrm{BM}]^{-1}\right)$ during break times (19 times) of a tennis match simulation attenuated the rise of Tre from the second half of exercise until the end of exercise, but exercise performance was not measured in this study.

Based on these previous studies, the magnitude of reduction of Tre may be affected by the ingestion volume of ice slurry. It may be possible to ingest a larger volume of ice slurry during HT or break times, but intake during exercise is unlikely. Nevertheless, it is possible that ingestion of ice slurry during half and break times is an effective method of attenuating the rise in core temperature to enhance exercise performance in repeated-sprint exercises.

Therefore, the purpose of this study was to investigate the effects of ice slurry ingestion during break times and the HT break on repeated sprint performance and core temperature in the heat. We hypothesized that the intermittent ingestion of ice slurry during break times would reduce the Tre in the second half of exercise, in turn improving repeated sprint performance.

\section{Materials and Methods}

\section{Participants}

Seven non-heat-acclimatized physically active males (age $=31 \pm 4$ years, height $=1.74 \pm 0.04 \mathrm{~m}, \mathrm{BM}=69.14 \pm 9.63 \mathrm{~kg}$ ) were recruited for this study. Participants completed a minimum of 6 hours of training per week at the time of study enrollment. All participants were non-smokers and normotensive; they did not have any known autonomic dysfunction and cardiovascular disease and did not take any medications. The study protocol was approved by the Ethics Committee of the Japan Institute of Sports Sciences (2018-007) in Japan. All participants provided written informed consent prior to commencing the study. The study complied with the latest version of the Declaration of Helsinki and was conducted according to international standards [13].

\section{Experimental procedures}

Before the experimental trials were completed, participants performed one familiarization trial with the same protocol as that in the experimental trial. The participants performed two different trials in a randomized cross-over design as follows: ingestion of ice slurry $\left(-1{ }^{\circ} \mathrm{C}\right.$ : ICE) or room temperature drink $\left(36.5^{\circ} \mathrm{C}\right.$ : CON $)$ at each break and HT. During all sessions, exercise was performed in a climate chamber to simulate the hot conditions at $36.5 \pm 0.5^{\circ} \mathrm{C}$ ambient temperature, $50 \pm 3 \%$ relative humidity. Each trial was separated by at least 7 days and commenced at the same time to control for circadian variations in body temperature. Throughout the study period, participants were asked to maintain their normal lifestyle, including their physical activity and nutritional habits. During the 24-hour period before the experimental trial, the participants were instructed to avoid strenuous exercise, as well as the consumption of alcohol, caffeine, nonsteroidal anti-inflammatory drugs, and nutritional supplements. Each participant arrived at the laboratory after having refrained from eating for 6 hours and drinking any beverage for 2 hours. They were instructed to drink $500 \mathrm{~mL}$ of plain water 2 hours before all tests to help promote hydration prior to the start of each trial.

\section{Repeated-sprint exercise}

- Figure 1 shows the experimental protocol. After the remaining instrumentation was affixed, participants entered the climate chamber. Participants performed 30 sets (1 block) of 1-min periods of repeated-sprint exercise protocol using a cycling ergometer (Fujin-Raijin; O.C.Labo, Tokyo, Japan). Each period consisted of 5 sec of maximal pedaling at the load of weight $\times 0.075(\mathrm{kp}), 25 \mathrm{sec}$ of pedaling with no-workload, and 30 sec of rest; two blocks of exercise periods were separated by $10 \mathrm{~min}$ of rest (HT). Each break was implemented for 1 min per every 5 sets. During the last $5 \mathrm{~s}$ of each passive recovery period, participants were instructed to assume a "ready" position, and a 3-s countdown was given prior to the start of each sprint. Cycling was performed in a seated position throughout the trial. Strong verbal encouragement was provided for every sprint effort. Participants completed a laboratory-based intermittent exercise protocol designed to replicate the demands of actual intermittent athletic games. One stage and quarter consisted of 5 sets and 15 sets, respectively; all participants performed a total of 4 quarters.

\section{Cooling intervention}

In ICE and CON trials, the participants ingested either $1.25 \mathrm{~g} \cdot \mathrm{kg} \mathrm{BM}^{-}$ 1 of ice slurry $\left(-1{ }^{\circ} \mathrm{C}\right)$ or room temperature drink $\left(36.5^{\circ} \mathrm{C}\right)$ throughout each break and $7.5 \mathrm{~g} \cdot \mathrm{kg} \mathrm{BM}^{-1}$ of a similar drink during HT. The total volume of ingestion was identical for CON and ICE. Ice slurry $\left(-1^{\circ} \mathrm{C}\right)$ was made using a slurry machine (Big Biz1; FMI, Osaka, Japan), and all drinks were a conventional sports drink (Pocari Sweat, Otsuka Pharmaceutical, Tokyo, Japan).

\section{Measurements and calculations}

Urine samples were measured to evaluate the hydration status by urine specific gravity (USG) before and after exercise, which was determined using a digital USG scale (PAL-09S; Atago, Tokyo, Japan). Nude BM was measured using a weighing scale (HW$100 \mathrm{KGV}, \mathrm{A} \& \mathrm{D}$, Tokyo, Japan) to the nearest $10 \mathrm{~g}$ before and after exercise. Throughout the two trials, participants self-inserted a rectal probe (ITP010-11, Nikkiso-Therm Co., Ltd., Tokyo, Japan) approximately $150 \mathrm{~mm}$ past the anal sphincter. Tre was continuously recorded via a data logger (N542R; Nikkiso-Therm Co., Ltd., Tokyo, 


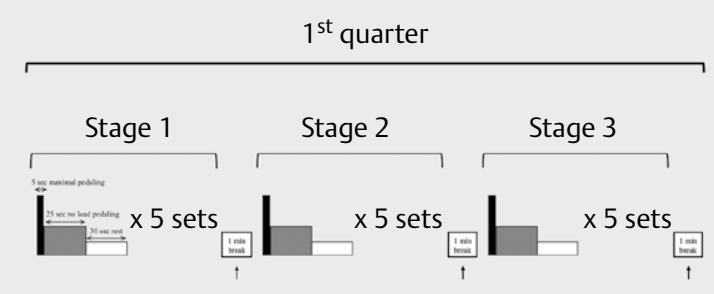

ICE or CON drink

$\uparrow\left(1.25 \mathrm{~g} \cdot \mathrm{kgBM}^{-1}\right)$ $\left(36.5^{\circ} \mathrm{C}, 50 \%\right.$ relative humidity)

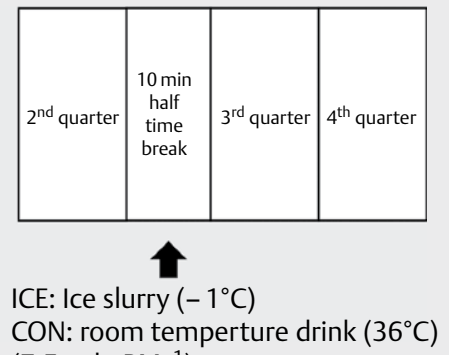

$\left(7.5 \mathrm{~g} \cdot \mathrm{kgBM}^{-1}\right)$

Fig 1 Schematic representation of the experimental protocol. Total of 30 sets of 1 -min periods of intermittent exercise by using a cycling ergometer, each period consisting of $5 \mathrm{sec}$ of maximal pedaling, $25 \mathrm{sec}$ of pedaling with no work load, and $30 \mathrm{sec}$ of rest. Each break was implemented 1 min every 5 sets. One stage and one quarter consisted of 5 sets and 15 sets, respectively. Two blocks of exercise periods were separated by 10 min of half-time break. Participants ingested ice slurry or room temperature drink during each break and half-time break. $\uparrow$ shows when participants ingested $1.25 \mathrm{~g} \cdot \mathrm{kg} \mathrm{BM}^{-1}$ of ice slurry or room temperature drink. $\downarrow$ shows when participants ingested $7.5 \mathrm{~g} \cdot \mathrm{kg} \mathrm{BM}^{-1}$ of ice slurry or room temperature drink.

Japan) and logged intermittently at 1 -min intervals. Four skin temperatures (forehead [Thead], chest, forearm, and thigh) were also recorded via iButtons (Thermocron Type SL; KN Laboratories Inc., Osaka, Japan) that were affixed using hypoallergenic polyacrylate adhesive tape. Onitsuka et al. [14] and Saldaris et al. [15] mentioned that Thead could be a useful index of brain temperature. The skin temperature (Tsk) was calculated using the following formula by Roberts et al. [16]: Tsk $=0.43 \times$ (chest temperature) $+0.25 \times$ (forearm temperature) $+0.32 \times$ (thigh temperature). The total sweat volume was calculated using the following formula: (BM before the experiment - BM after the experiment) + the amount of the ingested drink. A heart rate (HR) monitor (RS800; Polar, Lake Success, NY, USA) was strapped to the chest and recorded every 1 min throughout the trial. The physiological strain index (PSI) was calculated and then categorized from 0 (no strain) to 10 (very high strain) as described by Moran et al. [17], where Tre 0 and HRO are the initial Tre and heart rate, respectively, and where Tret and HRt are simultaneous measurements taken at any time. Power output and work done during each sprint were continuously recorded at a sampling rate of $10 \mathrm{~Hz}$ during maximal pedaling.

Ratings of the subjective thermal sensation [18] (TS; 9-point scale ranging from 1 = "very cold" to 9 = "very hot") and perceived exertion [19] (RPE; 15-point scale) were recorded at each break.

\section{Statistical analysis}

Descriptive data are presented as means \pm standard deviations (SD) and data were checked for normality. An a priori sample-size calculation (using an effect size of 0.6 , a power of 0.8 and alpha set to 0.05) was performed using $G$ * Power (version 3.1.9.2; Düsseldorf, Germany), and this indicated that we would need seven participants to find statistical significance. All statistical computations were performed using the IBM SPSS Statistics 24 software package (IBM Corp., Armonk, NY, USA). Two-way (drink $\times$ time) repeatedmeasures ANOVA was performed to compare the changes in the Tre, Tsk, Thead, peak power output, mean power output, and work done in each quarter (every 15 sets), HR, PSI, TS, RPE, BM, and USG between the experimental conditions. When a significant main ef- fect or interaction effect was identified, differences were delineated using a Bonferroni adjustment. When the sphericity assumption was violated, the Greenhouse-Geisser correction was employed. Total sweat volume, the rate of change in temperature, average of power output, and work done were compared using the paired t-test. For all comparisons, significance was set at a $p$ value of $<0.05$. Cohen's $d(d)$ was used as a measure of effect size for paired samples; a $d$ of 0.2 to $<0.5$ and $\geqq 0.5$ to $<0.8$ has been suggested to represent a small and medium treatment effect, respectively, whereas a $d \geqq 0.8$ represents a large treatment effect [20].

\section{Results}

\section{Hydration state}

The mean volume of drink consumed during the exercise period was $1389 \pm 195 \mathrm{~g}$ for all participants. The hydration state before and after the experiment is summarized in > Table $\mathbf{1}$. There were no significant differences in the pre-exercise measurements of BM (CON: $69.07 \pm 9.16 \mathrm{~kg}$, ICE: $69.31 \pm 9.50 \mathrm{~kg})$ and USG (CON: $1.013 \pm 0.009$, ICE: $1.015 \pm 0.008)$ between conditions $(p>0.05$, $d=0.03-0.24)$. When the effect of CON vs. ICE was compared, there was no effect on BM and USG for time or between trials. Total sweat volume was lower in the ICE trial compared to CON $(p=0.02$, $d=0.54)$.

\section{Rectal temperature (Tre)}

Mean baseline absolute Tre was similar between the CON $\left(37.09 \pm 0.24^{\circ} \mathrm{C}\right)$ and ICE trials $\left(37.25 \pm 0.29^{\circ} \mathrm{C}, p=0.30, d=0.60\right)$. However, Tre in the ICE trial was significantly lower than that in the CON trial at stage $9(p=0.04, d=1.00$; $>$ Fig. 2$)$. These differences in Tre persisted throughout the remainder of the experiment $(p<0.05, d=0.91-1.40)$. The changes in Tre ( $\Delta$ Tre) during the ICE trial was significantly lower than that during the CON trial beginning at stage $7(p=0.01, d=1.15)$. These differences in $\Delta$ Tre persisted throughout the remainder of the experiment $(p<0.05$, $d=1.19-1.83$ ). 
- Table 1 The hydration states before and after the experiment.

\begin{tabular}{|l|l|l|l|c|}
\hline \multirow{2}{*}{} & \multicolumn{2}{|c|}{ CON } & \multicolumn{2}{c|}{ ICE } \\
\cline { 2 - 5 } & Before & After & Before & After \\
\hline Body mass $(\mathrm{kg})$ & $69.07 \pm 9.16$ & $69.02 \pm 9.56$ & $69.31 \pm 9.50$ & $69.40 \pm 9.87$ \\
\hline Total sweat volume $(\mathrm{kg})$ & & & $1.291 \pm 0.240 *$ \\
\hline Urine specific gravity & $1.013 \pm 0.009$ & $1.432 \pm 0.281$ & $1.015 \pm 0.008$ & $1.016 \pm 0.007$ \\
\hline$*$ CON vs. ICE $(\mathrm{P}<0.05)$. & & & \\
\hline
\end{tabular}

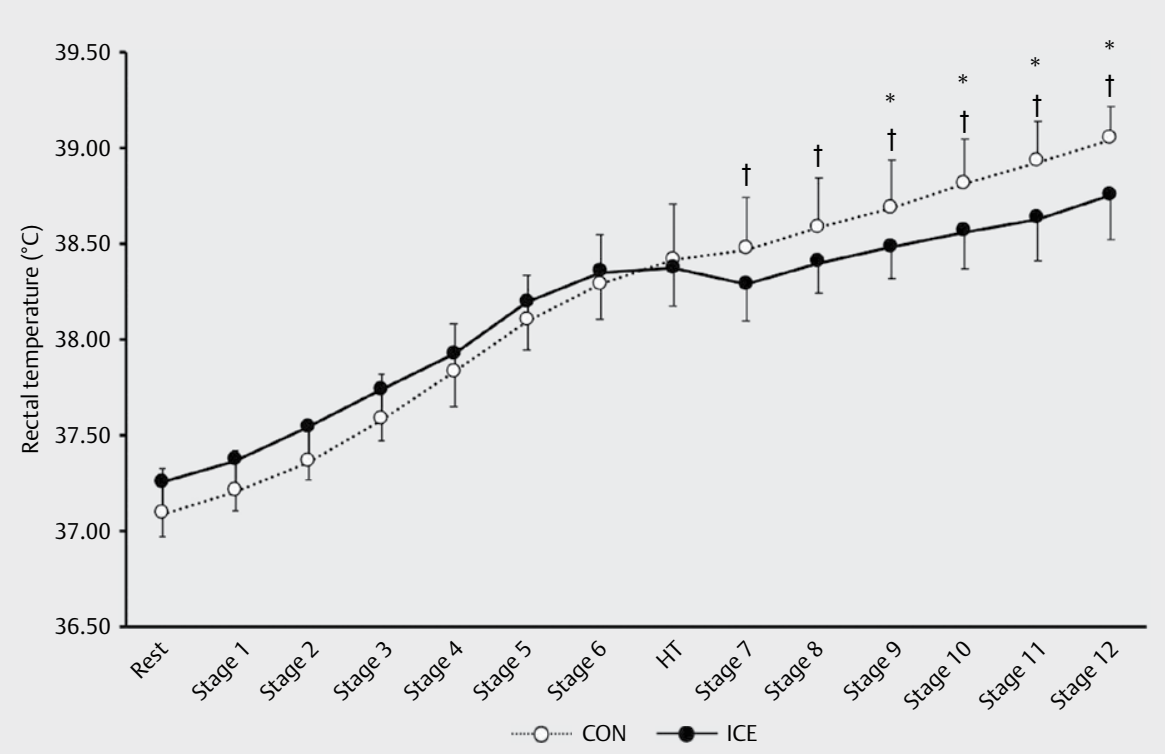

- Fig. 2 The rectal temperature during exercise under the two experimental conditions. The mean values are expressed as mean $\pm S D$ of seven participants (CON:O, ICE: $\bullet$ ). Time $\times$ drink effect CON vs. ICE: ${ }^{*} \mathrm{P}<0.05$ (absolute values), $\dagger \mathrm{P}<0.05$ (change rate values).

\section{Forehead skin temperature (Thead) and mean skin temperature (Tsk)}

The Thead was not significantly different between CON $\left(35.90 \pm 0.48^{\circ} \mathrm{C}\right)$ and ICE trials at rest $\left(35.77 \pm 0.33^{\circ} \mathrm{C}, p=0.47\right.$, $d=0.32$ ). Thead increased progressively in each trial until stage 6 , but there were no significant differences in Thead between trials ( $\triangleright$ Fig. 3a). The rate of change in the Thead at stage 6-12 (HT and second half of exercise) was lower in the ICE trial $\left(-0.27 \pm 0.41{ }^{\circ} \mathrm{C}\right)$ than in the CON trial $\left(0.10 \pm 0.31{ }^{\circ} \mathrm{C}, p=0.04\right.$, $d=1.02$ ). No significant differences in the Tsk were observed between trials at rest (CON: $34.33 \pm 0.41^{\circ} \mathrm{C}$ vs. ICE: $34.17 \pm 0.48^{\circ} \mathrm{C}$, $p=0.20, d=0.36$ ). Tsk increased progressively in each trial until stage 6, but Tsk in the ICE trial was significantly lower than that in the CON trial at HT $(p=0.04, d=1.11 ; \triangleright$ Fig. $3 \mathbf{b})$. These differences in Tsk persisted throughout the remainder of the experiment $(p<$ $0.05, d=0.89-0.94)$.

\section{Peak power output, mean power output, and work done in each quarter}

The peak power output, mean power output, and work done in each quarter are presented in $>$ Table 2 . Mean power output and peak power output were not significantly different between CON and ICE in each quarter $(p>0.05, d=0.04-0.41)$. However, the av- erage and total value of work done was lower in the ICE trial than that in the CON trial $(p<0.05, d=0.40-0.61)$.

\section{Heart rate (HR) and physiological strain index (PSI)}

There were no significant differences in HR between trials $(p>0.05$, $d=0.13-0.27)$. PSI was higher in the ICE trial before the HT break compared to that in the CON trial ( $p<0.05, d=0.45)$, but PSI during the second half of exercise was observed to be significantly lower than in the CON trial ( $p<0.05, d=0.50-0.54$; $\triangleright$ Table 3).

\section{Ratings of thermal sensation (TS) and perceived exertion (RPE)}

Although TS was significantly lower in the ICE trial than in the CON trial at The HT break ( $p=0.03, d=1.20$; $>$ Table 3), there were no significant differences at other points. RPE in the ICE trial was significantly lower in stage 12 compared with the CON trial $(p=0.02$, $d=0.91)$.

\section{Discussion}

To the best of our knowledge, this is the first study to compare the thermoregulatory responses and repeated-sprint performance resulting from ice slurry (ICE) and room temperature drink (CON) in- 

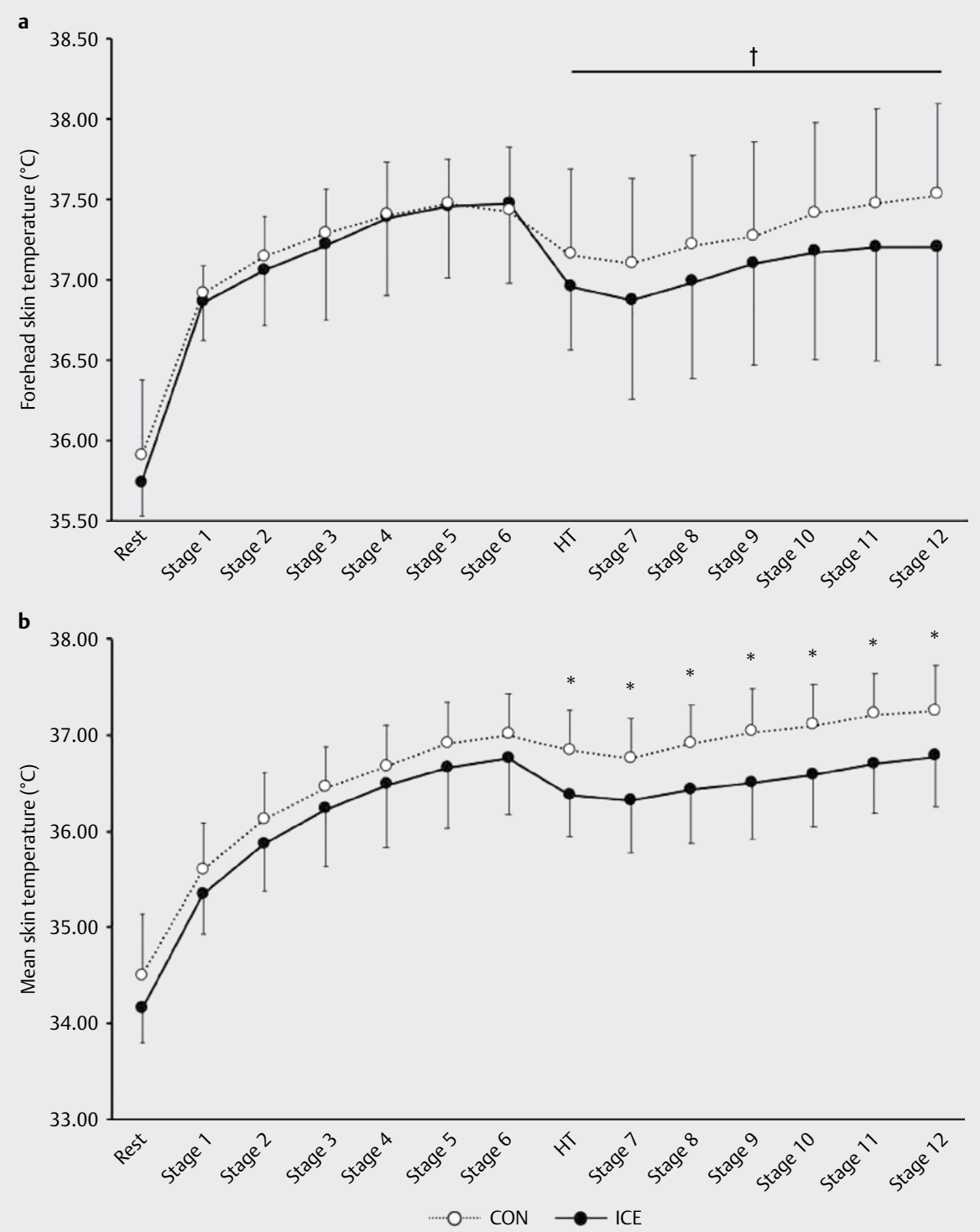

- Fig. 3 The forehead skin temperature $\mathbf{a}$ and mean skin temperature $\mathbf{b}$ during exercise under the two experimental conditions. The mean values are expressed as mean \pm SD of seven participants (CON: $\odot, I C E: \bullet)$. Time $\times$ drink effect CON vs. ICE: ${ }^{*} \mathrm{P}<0.05$ (absolute values), $† \mathrm{P}<0.05$ (change rate values).

gestion during break times and the HT break in hot conditions. In accordance with our hypothesis, ice slurry ingestion during break times and the HT break was significantly more effective at attenuating the increase in Tre than was room temperature drink ingestion after stage 9 of repeated-sprint exercises in the heat. Although ice slurry ingestion during break times and the HT break did not improve the peak power output and mean power output in the second half of exercise, the total and average values of work done were enhanced in the ICE trial compared to those in the CON trial. The other important findings were as follows: Tsk, Thead, total sweat volume, PSI, and perception in the ICE trial were significantly lower than those in the CON trial.
The present study showed the effectiveness of ice slurry ingestion during break times and HT break, but ice slurry ingestion during the first half of exercise did not attenuate the rise of Tre. This result is consistent with the findings of a previous study that did not show cooling by conduction during the first half of exercise [21]. In addition, Onitsuka et al. [21] also showed that ingestion of $7.5 \mathrm{~g} \cdot \mathrm{kg} \mathrm{BM}^{-1}$ of ice slurry during $\mathrm{HT}$ could reduce in Tre only until 10 min after $H$ T. In the present study, $\Delta$ Tre during the ICE trial was significantly lower than during the CON trial beginning at stage 7 , and the differences persisted throughout the remainder of the experiment. Based on these findings, ice slurry ingestion at HT and break times during the second half of exercise may be a more ac- 
- Table 2 Performance variables of each 15 sets (quarter) of repeated-sprint exercise

\begin{tabular}{|c|c|c|c|c|c|c|c|}
\hline & & 1st Quar & 2nd Quar & 3rd Quar & 4th Quar & Average & Total \\
\hline \multirow[t]{2}{*}{ PPO (W) } & $\mathrm{CON}$ & $826 \pm 81$ & $828 \pm 86$ & $805 \pm 83$ & $825 \pm 100$ & $818 \pm 79$ & \\
\hline & ICE & $836 \pm 80$ & $831 \pm 55$ & $809 \pm 54$ & $836 \pm 55$ & $838 \pm 73$ & \\
\hline \multirow[t]{2}{*}{$\mathrm{PPO}(\mathrm{W} / \mathrm{kg})$} & CON & $12.0 \pm 0.9$ & $12.1 \pm 1.2$ & $11.7 \pm 1.4$ & $12.0 \pm 1.6$ & $12.0 \pm 1.2$ & \\
\hline & ICE & $12.9 \pm 1.9$ & $13.0 \pm 0.9$ & $12.6 \pm 1.9$ & $13.0 \pm 1.9$ & $13.0 \pm 2.0$ & \\
\hline \multirow[t]{2}{*}{ MPO (W) } & CON & $692 \pm 54$ & $682 \pm 57$ & $654 \pm 51$ & $670 \pm 64$ & $674 \pm 56$ & \\
\hline & ICE & $699 \pm 58$ & $695 \pm 49$ & $679 \pm 48$ & $695 \pm 58$ & $692 \pm 53$ & \\
\hline \multirow[t]{2}{*}{ MPO (W/kg) } & CON & $10.1 \pm 0.9$ & $9.9 \pm 1.0$ & $9.6 \pm 1.2$ & $9.8 \pm 1.3$ & $9.8 \pm 1.1$ & \\
\hline & ICE & $10.2 \pm 0.8$ & $10.1 \pm 0.9$ & $9.9 \pm 0.9$ & $10.1 \pm 1.0$ & $10.1 \pm 0.9$ & \\
\hline \multirow[t]{2}{*}{ WORK (kJ) } & $\mathrm{CON}$ & $16.5 \pm 1.5$ & $16.7 \pm 1.2$ & $16.1 \pm 1.0$ & $16.4 \pm 1.2$ & $16.4 \pm 1.2$ & $196.9 \pm 12.6$ \\
\hline & ICE & $16.9 \pm 1.4$ & $17.4 \pm 1.3$ & $16.9 \pm 1.3$ & $17.3 \pm 1.7$ & $17.1 \pm 1.4$ & $205.7 \pm 16.2^{*}$ \\
\hline \multirow[t]{2}{*}{ WORK $(\mathrm{J} / \mathrm{kg})$} & CON & $240 \pm 28$ & $243 \pm 26$ & $236 \pm 30$ & $240 \pm 31$ & $240 \pm 28$ & $959 \pm 110$ \\
\hline & ICE & $246 \pm 23$ & $253 \pm 25$ & $247 \pm 25$ & $252 \pm 21$ & $250 \pm 23$ & $999 \pm 91^{*}$ \\
\hline
\end{tabular}

Table 3 Perceptual sensation, HR, and PSI during experiment.

\begin{tabular}{|c|c|c|c|c|c|c|c|}
\hline & & Rest & Stage 3 & Stage 6 & HT & Stage 9 & Stage 12 \\
\hline \multirow[t]{2}{*}{ HR (bpm) } & CON & $79.7 \pm 8.0$ & $132.3 \pm 18.0$ & $147.0 \pm 17.2$ & $116.0 \pm 14.9$ & $153.0 \pm 16.1$ & $159.0 \pm 18.0$ \\
\hline & ICE & $81.0 \pm 11.4$ & $144.0 \pm 13.0$ & $158.9 \pm 14.8$ & $114.1 \pm 10.1$ & $156.1 \pm 14.2$ & $164.1 \pm 19.4$ \\
\hline \multirow[t]{2}{*}{ PSI } & CON & $0.0 \pm 0.0$ & $3.66 \pm 0.82$ & $5.86 \pm 1.07$ & $4.57 \pm 0.87$ & $6.98 \pm 1.01$ & $8.06 \pm 0.98$ \\
\hline & ICE & $0.0 \pm 0.0$ & $4.24 \pm 0.82$ & $6.34 \pm 1.07^{*}$ & $4.12 \pm 0.37$ & $6.49 \pm 0.80^{*}$ & $7.50 \pm 1.23 *$ \\
\hline \multirow[t]{2}{*}{ TS } & CON & $7.4 \pm 0.5$ & $8.3 \pm 0.8$ & $8.3 \pm 0.5$ & $7.6 \pm 1.4$ & $8.3 \pm 0.5$ & $8.3 \pm 0.5$ \\
\hline & ICE & $7.4 \pm 0.7$ & $8.0 \pm 0.6$ & $8.3 \pm 0.5$ & $6.3 \pm 1.6^{*}$ & $8.3 \pm 0.5$ & $8.3 \pm 0.5$ \\
\hline \multirow[t]{2}{*}{ TC } & CON & $2.6 \pm 1.0$ & $1.7 \pm 0.8$ & $1.6 \pm 0.5$ & $2.4 \pm 1.4$ & $1.4 \pm 0.5$ & $1.3 \pm 0.5$ \\
\hline & ICE & $2.8 \pm 1.0$ & $1.9 \pm 0.9$ & $1.6 \pm 1.5$ & $3.3 \pm 1.7^{*}$ & $1.6 \pm 0.5$ & $1.6 \pm 0.5$ \\
\hline \multirow[t]{2}{*}{ RPE } & CON & & $15.7 \pm 1.3$ & $16.7 \pm 1.9$ & & $17.4 \pm 1.3$ & $18.1 \pm 1.5$ \\
\hline & ICE & & $15.7 \pm 2.2$ & $16.3 \pm 2.1$ & & $17.1 \pm 1.2$ & $17.1 \pm 1.3^{*}$ \\
\hline
\end{tabular}

ceptable strategy for cooling to maintain a lower Tre with less discomfort during exercise in the heat.

In the present study, the improved total and average work done demonstrated in ice slurry ingestion may be primarily because of the lower Tre associated with this condition throughout the second half of exercise. Castel et al. [22] showed that the total work performed during repeated-sprint performance was significantly higher after using a cooling vest compared to no cooling trial. Brade et al. [23] also demonstrated that ingestion of ice slurry and using a cooling jacket during pre-exercise and HT enhanced the total work done. Both previous studies observed that the reduction of core temperature coincides with a performance improvement. Additionally, ice slurry ingestion during break times and HT break also reduced Tsk compared to that of a room temperature drink. Schlader et al. [24] reported that intermittent exercise performance was improved without changes in Tre by a lower Tsk. Naito and Ogaki [25] noted that the rate of rise in the Tsk during exercise was significantly slower after ingestion of crushed ice. A lower Tsk results in a higher core-to-skin temperature gradient, leading to a slower rise in the Tre during exercise [1]. Therefore, it is possible that the reductions of Tre and Tsk positively influenced improvements of total and average work done.
The other potential explanation for the improvement of total and average work done may be explained by the reduction of perceptual sensations. In this study, TS or RPE was temporarily improved in the ICE trial compared to that of CON trial. Some studies suggested that perceptual sensations were affected by cold receptors located in skin, mouth, and gastrointestinal tract, which might stimulate a greater density of cold-sensitive thermal afferents to the hypothalamus. Moreover, it is possible that improvements in perceptual sensations are due to the rise of Tsk [24, 26] and Thead [14]. Conductive cooling by oral ingestion of ice slurry may allow direct attenuation of the rise of Thead [12] and brain temperature [27] measured by magnetic resonance spectroscopy. Therefore, it is speculated that the improvements of perceptual sensation using ice slurry ingestion were influenced by cold-sensitive thermal afferents, from the peripheral to the central, and attenuated both peripheral and brain temperatures.

The development of total sweat volume during exercise in hot conditions induces dehydration, which causes a significant increase in cardiovascular strain, such as a reduction of plasma volume or blood flow to the skin. In this study, the total sweat volume was lower in the ICE trial than that in the CON trial. This finding is consistent with previous studies, which reported that the use of ice 
slurry ingestion during exercise reduced total sweat volume compared to ingestion of cold water [12]. However, it is speculated that the reduction of total sweat volume may appear to decrease the potential for evaporative heat loss from the skin. Jay and Morris [28] noted that this might not necessarily result in a net cooling effect. This cooling effect could be due to a reduction in sweating that lowers the evaporative heat loss from the skin by an amount that is at least equivalent to the additional internal heat loss with the ingested fluid. Indeed, Morris et al. [29] calculated that a higher evaporative heat loss by warm drink ingestion provided a large net heat loss compared to ice slurry ingestion. However, in a previous review, Jay and Morris [28] mentioned that a net cooling effect with ice slurry ingestion depends on the combinations of exercise types and climate conditions; internal heat loss is $100 \%$ efficient in humid conditions, such as the environment of this study, because not all secreted sweat evaporates and contributes to cooling. Additionally, attenuating the rise of Tre was observed in the ICE trial in this study, contrary to the above previous study [29]. Therefore, it is speculated that a larger internal heat loss with ice slurry ingestion, rather than an increase of evaporative heat loss by ingestion of room temperature liquid, may have contributed to the net heat loss in this study, thereby attenuating the rise of Tre in hot and humid conditions.

Greater heat strain during exercise in the heat leads to both impairment of exercise performance and heat injury associated with hyperthermia. Buller et al. [30] described a PSI value of 7.5 or more as the "at risk" category for heat-related illness. Although HR did not decrease in the ICE trial, by attenuating the strain in Tre during the second half of exercise following ice slurry ingestion, participants kept the PSI level below the critical marker of 7.5 throughout the experiment. By lowering the risk for heat-related illness, participants in the ICE trial may be able to sustain workloads at a high level, thereby improving total and average work done.

This study indicated the advantage of more options for cooling strategy in sports competitions. However, it may be necessary to ingest a somewhat large amount of ice slurry during the HT period to reduce heat stress. If athletes hope to reduce core temperature during the first half of exercise, they should select ice slurry ingestion before exercise. Pre-cooling has been proposed to minimize exercise performance impairments and prevent hyperthermia. Naito and Ogaki [31] demonstrated that pre-cooling by intermittent ice ingestion for a 30-min period decreased Tre on resting compared with that of cold water ingestion. Recently, Aldous et al. [32] showed that combined cooling (Pre $+\mathrm{HT}$ ) augmented physical performance during an intermittent soccer performance test compared to that of a no-cooling trial. Further research is warranted to compare the effects of the different timing of cooling strategies, such as pre-cooling, HT, break time cooling or combined cooling, by intermittent ice slurry ingestion.

\section{Limitations}

The small sample size of the present study is a limitation, but total and mean work done was statistically improved. Further, research is warranted with a larger sample size to further elucidate the mechanisms for the performance enhancement. Peak power output and mean power output showed approximately $2.5-8 \%$ improvement in the ICE trial but no statistically significant differences between ICE and CON trials. This might have been due to the large difference in physical fitness levels of participants. In this study, there were differences of approximately $250 \mathrm{~W}$ in peak power output and approximately $200 \mathrm{~W}$ in mean power output, respectively, between the highest and lowest participants. Previous studies demonstrated that HT cooling attenuated the rise of Tre at the second half of exercise. Nevertheless, there were no significant differences in peak power output or mean power output between cooling and no-cooling trials [21,33]. Notably, Maroni et al. [33] reported that the standard deviation in peak power output value was approximately $15 \%$ of the mean value. Second, we did not conduct a cold-water ingestion trial. However, sports involving repeated-sprint exercises with water break times, such as soccer or rugby, often involve ingesting drinks from bottles lying on the ground. Bottle drinks are warmed to the same level as the ambient temperature if athletes are exercising in hot conditions. Therefore, we selected room-temperature drink ingestion as the control trial. In contrast, cold water or ice slurry is usually available for ingestion during $\mathrm{HT}$ breaks in locker rooms or at the bench. During HT, participants ingested the same drink as at break times, since the primary aim was to assess the influence of ice slurry ingestion on thermal strain and exercise performance compared to room temperature water ingestion. Finally, in this protocol, the exercise task of active recovery by pedaling with no workload was not controlled. It is possible that exercise intensity in the active recovery period may have influenced subsequent main performance. However, athletes in actual competition show decreased performance, such as jogging, in the second half of exercise [34]. Further research should be conducted to investigate a controlled protocol during active recovery to evaluate the main performance.

\section{Conclusions}

Ice slurry ingestion during break times and the HT break significantly attenuated the increase in Tre, Tsk, and Thead compared with room temperature drink ingestion at the second half of exercise (during repeated-sprint exercises), thereby improving total and mean work done in the heat. This study indicates that a sufficient amount of ice slurry ingestion during break times and HT break may be a more practical and effective cooling strategy to attenuate the rise of core temperature in the second half of exercise and to improve the repeated-sprint exercise capacity in the heat. Further research is necessary to validate the effects of the timing of the cooling maneuver, such as pre-cooling, $\mathrm{HT}$, break time cooling, or combined cooling, by intermittent ice slurry ingestion.

\section{Acknowledgements}

The authors thank the volunteers who donated their time and effort to participate in the present study.

\section{Conflict of Interest}

The authors declare that they have no conflict of interest. 


\section{References}

[1] Siegel R, Mate ], Watson G, Nosaka K, Laursen PB. Pre-cooling with ice slurry ingestion leads to similar run times to exhaustion in the heat as cold water immersion. J Sports Sci 2012; 30: 155-165

[2] Ihsan M, Landers G, Brearley M, Peeling P. Beneficial effects of ice ingestion as a precooling strategy on 40-km cycling time-trial performance. Int J Sports Physiol Perform 2010; 5: 140-151

[3] Naito T, Iribe $\mathrm{Y}$, Ogaki T. Ice ingestion with a long rest interval increases the endurance exercise capacity and reduces the core temperature in the heat. J Physiol Anthropol 2017; 36: 9

[4] Marino FE. Methods, advantages, and limitations of body cooling for exercise performance. $\mathrm{Br}$ J Sports Med 2002; 36: 89-94

[5] Quod M], Martin DT, Laursen PB. Cooling athletes before competition in the heat: comparison of techniques and practical considerations. Sports Med 2006; 36: 671-682

[6] Siegel R, Laursen PB. Keep your cool: possible mechanisms for enhanced exercise performance in the heat with internal cooling methods. Sports Med 2012; 42: 89-98

[7] Siegel R, Mate J, Brearley MB, Watson G, Nosaka K, Laursen PB. Ice slurry ingestion increases core temperature capacity and running time in the heat. Med Sci Sports Exerc 2010; 42: 717-725

[8] Stevens C], Dascombe B, Boyko A, Sculley D, Callister R. Ice slurry ingestion during cycling improves Olympic distance triathlon performance in the heat. J Sports Sci 2013; 31: 1271-1279

[9] Adams WB, Mcdermott BP, Schmit C, Kenny GP. Body cooling. In: Casa DJ, Ed. Sport and Physical Activity in the Heat - Maximizing Performance and Safety. Switzerland: Springer; 2019: 59-81

[10] Maunder E, Laursen PB, Kilding AE. Effect of ad libitum ice-slurry and cold-fluid ingestion on cycling time-trial performance in the heat. Int J Sports Physiol Perform 2017; 12: 99-105

[11] Stanley J, Leveritt M, Peake JM. Thermoregulatory responses to ice-slush drink ingestion and exercise in the heat. Eur J Appl Physiol 2010; 110: 1163-1173

[12] Naito T, Sagayama H, Akazawa N, Haramura M, Tasaki M, Takahashi H. Ice slurry ingestion during break times attenuates the increase of core temperature in a simulation of physical demand of match-play tennis in the heat. Temperature (Austin) 2018; 5: 371-379

[13] Harriss D], Macsween A, Atkinson G. Standards for ethics in sport and exercise science research: 2018 update. Int J Sports Med 2017; 38: 1126-1131

[14] Onitsuka S, Zheng X, Hasegawa $H$. Ice slurry ingestion reduces both core and facial skin temperatures in a warm environment. J Therm Biol 2015; 51: 105-109

[15] Saldaris JM, Landers G], Lay BS, Zimmermann MR. Internal precooling decreases forehead and core temperature but does not alter choice reaction time during steady state exercise in hot, humid conditions. J Therm Biol 2019; 81: 66-72

[16] Roberts MF, Wenger CB, Stolwijk JA, Nadel ER. Skin blood flow and sweating changes following exercise training and heat acclimation. J Appl Physiol 1977; 43: 133-137

[17] Moran DS, Shitzer A, Pandolf KB. A physiological strain index to evaluate heat stress. Am J Physiol 1998; 275: R129-R134
[18] Kashimura O. Changes in thermal sensation during endurance exercise. J Phys Fitness. Sports Med 1986; 35: 264-269

[19] Borg GA. Psychophysical bases of perceived exertion. Med Sci Sports Exerc 1982; 14: 377-381

[20] Cohen J. Statistical Power Analysis for the Behavioral Sciences. 2nd ed. Hillsdale (NJ): Lawrence Erlbaum Associates 1988; 567p

[21] Onitsuka S, Ueno T, Zheng X, Hasegawa H. Effect of ice slurry ingestion during half-time breaks on intermittent exercise capacity and thermoregulation in the warm environment. Gazz Med Ital. Arch Sci Med 2015; 174: 113-121

[22] Castle PC, Macdonald AL, Philp A, Webborn A, Watt PW, Makwell NS. Precooling leg muscle improves intermittent sprint exercise performance in hot, humid conditions. J Appl Physiol 2006; 100: 1377-1384

[23] Brade C, Dawson B, Wallman K. Effects of different precooling techniques on repeat sprint ability in team sport athletes. Eur J Sport Sci 2014; 14: S84-S91

[24] Schlader Z], Simmons SE, Stannard SR, Mundel T. The independent roles of temperature and thermal perception in the control of human thermoregulatory behavior. Physiol Behav 2011; 103: 217-224

[25] Naito T, Ogaki T. Comparison of the effects of cold water and ice ingestion on endurance cycling capacity in the heat. J Sport Health Sci 2017; 6: 111-117

[26] Soo J, Tang G, Arjunan SP, Pang J, Aziz AR, Ihsan M. The effects of lower body passive heating combined with mixed-method cooling during half-time on second-half intermittent sprint performance in the heat. Eur J Appl Physiol 2019; 119: 1885-1899

[27] Onitsuka S, Nakamura D, Onishi T, Arimitsu T, Takahashi H, Hasegawa $\mathrm{H}$. Ice slurry ingestion reduces human brain temperature measured using non-invasive magnetic resonance spectroscopy. Sci Rep 2018; 8: 2757

[28] Jay O, Morris NB. Does cold water or ice slurry ingestion during exercise elicit a net body cooling effect in the heat? Sports Med 2018; 48: $17-29$

[29] Morris NB, Coombs G, Jay O. Ice slurry ingestion leads to a lower net heat loss during exercise in the heat. Med Sci Sports Exerc 2016; 48: 114-122

[30] Buller M], Latzka WA, Yokota M, Tharion W], Moran DS. A real-time heat strain risk classifier using heart rate and skin temperature. Physiol Meas 2008; 29: N79-N85

[31] Naito T, Ogaki T. Pre-cooling with intermittent ice ingestion lowers the core temperature in a hot environment as compared with the ingestion of a single bolus. J Therm Biol 2016; 59: 13-17

[32] Aldous JWF, Chrismas BCR, Akubat I, Stringer CA, Abt G, Taylor L. Mixed-methods pre-match cooling improves simulated soccer performance in the heat. Eur J Sport Sci 2019; 19: 156-165

[33] Maroni T, Dawson B, Dennis M, Naylor L, Brade C, Wallman K. Effects of half-time cooling using a cooling glove and jacket on manual dexterity and repeated-sprint performance in heat. J Sports Sci Med 2018; 17: 485-491

[34] Ozgunen KT, Kurdak SS, Maughan RJ, Zeren C, Korkmaz S, Yazici Z, Shirreffs SM, Binnet MS, Dvorak J. Effect of hot environmental conditions on physical activity patterns and temperature response of football players. Scand J Med Sci Sports 2010; 20: 140-147 